\title{
Transition from Quantum Hall to Compressible States in the Second Landau Level: New Light on the $\nu=5 / 2$ Enigma
}

\author{
R. H. Morf \\ Paul Scherrer Institute, Villigen, Switzerland
}

(Received 7 October 1997)

\begin{abstract}
Quantum Hall states at filling fraction $\nu=5 / 2$ are examined by numerical diagonalization. Spinpolarized and spin-unpolarized states of systems with $N \leq 18$ electrons are studied, neglecting effects of Landau level mixing. We find that the ground state is spin polarized. It is incompressible and has a large overlap with paired states like the Pfaffian. For a given sample, the energy gap is about 11 times smaller than at $\nu=1 / 3$. Evidence is presented of phase transitions to compressible states, driven by the interaction strength at short distance. A reinterpretation of experiments is suggested. [S0031-9007(97)05250-2]
\end{abstract}

PACS numbers: 73.40.Hm, 02.60.Dc, 02.70.-c

Ten years after the discovery of a quantized Hall plateau at filling fraction $\nu=5 / 2$ by Willett et al. [1], "a key piece of the $\nu=5 / 2$ puzzle is still missing": This is the conclusion reached by Eisenstein in his recent review [2,3]. Studies by Eisenstein et al. [4] in a tilted magnetic field had shown that the plateau disappears when the tilt angle exceeds a critical value. It is now widely believed that the plateau is the result of a spin-unpolarized incompressible ground state (GS), while, at larger tilt angles, the Zeeman energy favors a polarized compressible GS, consistent with the disappearance of the plateau.

The evidence supporting the above picture is taken from activation studies which reveal an energy gap that decreases with increasing tilt angle [5]. This fact is explained naturally if the GS is unpolarized and if its lowest energy excitations involve electrons with reversed spin, and thus a gain in Zeeman energy $\Delta E=g \mu_{B} B$ from spin reversal ( $g$ and $\mu_{B}$ stand for the $g$ factor and the Bohr magneton). This energy gain increases with increasing tilt angle $\Theta$ as the magnetic field perpendicular to the sample, $B_{\perp}=B \cos \Theta$, is fixed by the electron density $n_{S}$ of the sample and the filling fraction $\nu$ [6]. From the slope of the activation energy as a function of $B$, a $g$ factor $g \approx$ 0.56 was extracted $[3,5]$, somewhat larger than its value $g=0.44$ for bulk GaAs. That the polarized state expected at large tilt angles should be compressible is consistent with the fermion Chern-Simons theory of Halperin, Lee, and Read [7], which predicts that electrons in a halffilled Landau level (LL) behave like quasiparticles in zero magnetic field forming a Fermi liquid, the "composite fermion (CF) liquid" [8].

In this Letter, we challenge this interpretation of the experiments. We present evidence from exact diagonalization results that the GS in a half-filled second LL is spin polarized and incompressible, consistent with the prediction by d'Ambrumenil and the author [9] that the CF liquid does not form at this filling.

What makes the plateau disappear at large tilt angles? If the system is spin polarized already at small tilt angles, the Zeeman energy cannot drive the phase transition. In this Letter, we show that the incompressible state is very sensitive to details of the interaction: phase transitions to gapless states occur when the interaction at short distance is either "too hard" or "too soft." When it is too hard, we recover the compressible CF liquid as GS. We maintain that the system becomes gapless due to a phase transition to a compressible state, driven by tilting the magnetic field, thereby modifying the interaction.

In the following, we examine both spin-polarized and unpolarized systems by exact diagonalization [10]. We employ Haldane's spherical geometry [11], in which quantized Hall states at filling fraction $\nu_{n}$ of the $n$th LL are characterized by a specific relation between the number of electrons $N$ and the number of flux quanta $N_{\Phi}$

$$
N_{\Phi}=\nu_{n}^{-1} N-S \text {. }
$$

Here, the "shift" $S$ depends on $\nu_{n}$ and the character of the fractional quantum Hall $(\mathrm{FQH})$ state [12], and represents a topological quantum number [13]. Its value for the $\mathrm{FQH}$ state at $\nu=5 / 2=2+1 / 2, \nu_{1}=1 / 2$, is not known although definite predictions exist [14-16]. To locate the $\mathrm{FQH}$ state, we make an unbiased study for a whole range of $S$ values. We neglect LL mixing and approximate the electron interaction by the Coulomb interaction of point particles [17]. As usual, the interaction is specified by the values of Haldane's pseudopotentials [11] $V_{L}$, i.e., the interaction energy of two electrons with relative angular momentum $L$, which is LL dependent.

Results of our exact diagonalizations are shown in Fig. 1 [18]. Energies per electron $E / N$ for spin-polarized and spin-unpolarized systems at $\nu_{1}=1 / 2$, i.e., $N_{\Phi}=2 N-$ $S$, are shown for different $S$. Figure 1(a) shows results for unpolarized and Fig. 1(b) those for polarized systems. In Fig. 1(c), we show the difference between GS energies of unpolarized and polarized states. Energies are quoted in units $e^{2} / l_{0}$ where $l_{0}$ denotes the magnetic length, $l_{0}=$ $\sqrt{\hbar c / e B} ;$ cf. [19]. For even values of the flux $N_{\Phi}$, the GS of the unpolarized systems has angular momentum $L=$ 0 in all cases studied; cf. Fig. 1(a). As incompressible states must be rotation invariant in the spherical geometry, 


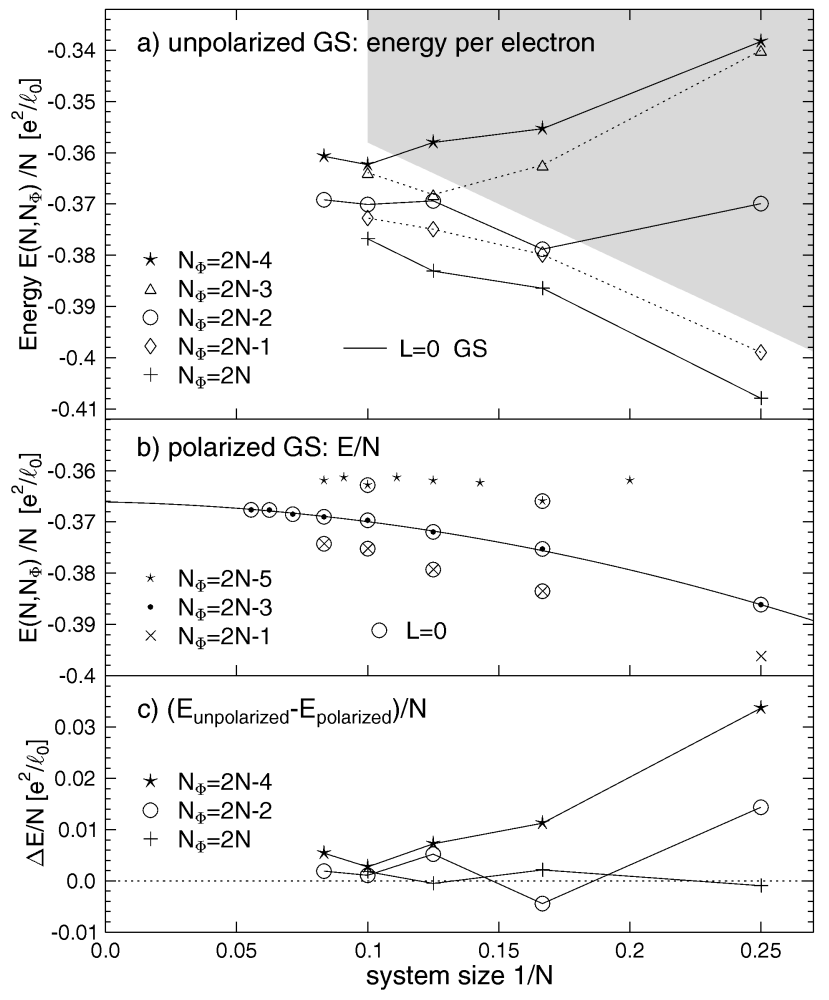

FIG. 1. For systems with $4 \leq N \leq 18$ electrons and flux $N_{\Phi}=2 N-S$ GS energies $E / N$ are shown for unpolarized and polarized systems. (a) Unpolarized system: $E / N$ for $S=$ $0,1,2,3,4$. At even $N_{\Phi}$, GS have angular momentum $L=0$. Results in the shaded area suffer from strong finite size effects; cf. text. (b) $E / N$ at $S=1,3,5$ for polarized systems. At $S=3$ and even $N$, i.e., the quantum numbers of the Pfaffian, all GS have angular momentum $L=0$. (c) Energy difference between unpolarized and polarized state for the same $N, N_{\Phi}$.

they are, at least in principle, candidates for $\mathrm{FQH}$ states. Yet, they have in almost all cases higher energy than polarized states at the same $N, N_{\Phi}$; cf. Fig. 1(c). The exception is the unpolarized state at $N=6, N_{\Phi}=10$ [see Fig. 1(c)]. At first, before investigating larger systems, we were hopeful that this observation might help to explain the $\nu=5 / 2$ Hall plateau. Our larger system studies do not support this hope: For systems with up to $N=12$ electrons, no similar unpolarized state exists, and there is no hint that in the bulk limit the GS would be unpolarized [20].

In fact, there is evidence that the properties of the GS at $N=6, N_{\Phi}=10$ are not related to $\nu=5 / 2$ : Similar "cusps" in $E / N$ occur at $N=8, N_{\Phi}=13$ and $N=10$, $N_{\Phi}=16$; cf. Fig. 1(a). These appear on the line $N_{\Phi}=$ $3 N / 2+1$, which extrapolates to $\nu_{1}=2 / 3$ for large $N$, and have nothing to do with the behavior at $\nu_{1}=1 / 2$. We believe that the cusps reflect a property that for values of $N_{\Phi}$ below this line [corresponding to the shaded area of Fig. 1(a)] it seems to be impossible to construct a spinsinglet wave function (wf) for which the pair correlation function $g(R)$ vanishes at $R=0$, whereas it is possible to do so for $N_{\Phi}$ on or above this line [21]. At filling $\nu_{1}=$
$1 / 2, N_{\Phi}=2 N-S$ exceeds this limit for large enough $N$. For smaller $N$, the GS have anomalously high energy; cf. shaded area in Fig. 1(a).

To complement this picture, we show in Fig. 2 the pair correlation function $g(R)$ for the unpolarized GS at $N=12, N_{\Phi}=22$, and its components $g_{\text {up-down }}(R)$ and $g_{\text {up-up }}(R)$, for electrons with unlike and like spins, respectively. Clearly, $g(R)$ is close to zero as $R$ tends to zero. In an unpolarized state, the number of electrons contributing to $g_{\text {up-down }}(R)$ is $N / 2$, while for $g_{\text {up-up }}(R)$ it is one less. In a local spin singlet, this extra electron is close to the electron at the origin [14], while in our state, it is as far away as possible on a sphere; see Fig. 2. A system whose GS is polarized, but in which a long-wavelength spin excitation establishes spin-singlet symmetry, would show such behavior.

In Fig. 1(b), the GS energy $E / N$ of polarized states is shown for systems with $N_{\Phi}=2 N-S$, at $S=1,3$, and 5. GS with $L=0$ are marked with circles. For even $S$, GS have typically $L>0$ and are not shown. Only for $S=3$, all GS for even $N$ are rotation invariant and thus candidates for $\mathrm{FQH}$ states. Their energy increases smoothly with size, extrapolating to a bulk limit of $\approx-0.366$. Their flux agrees with predictions based on pair formation $[15,16]$.

In Fig. 3(a), we show the energy spectrum of a $N=8$ electron system for different pair interactions, by varying the coupling strength $V_{1}$ in the $L_{\mathrm{rel}}=1$ relative angular momentum channel, but keeping all the other $V_{L}$ at their values for Coulomb interaction in the second LL. As we can see, around $V_{1}=1$ (in units of $V_{1}^{\text {Coulomb }}$ ) there is a gap $\Delta$ in the excitation spectrum $\Delta \approx 0.02$. However, for both small and large $V_{1}$, the gap disappears.

In Fig. 3(b) we show the overlap of the GS with the Pfaffian wf. Clearly, the overlap is close to unity when $V_{1}$ has the value for Coulomb interaction. In fact, overlap and gap have their maxima roughly at the same value $V_{1} \approx 1.1$. These results are consistent with conjectures by Greiter et al. [22] that the $\nu=5 / 2 \mathrm{FQH}$ state might be related to the Pfaffian. However, this observation should not be overstated: Indeed, the GS has a similarly large overlap with a pair wf [19], setting parameters $m=1, t=0, s=$ 2 in Eq. (1) of Ref. [19]. In view of the ambiguity of trial wf's, we cannot be sure that in the bulk limit, the GS will

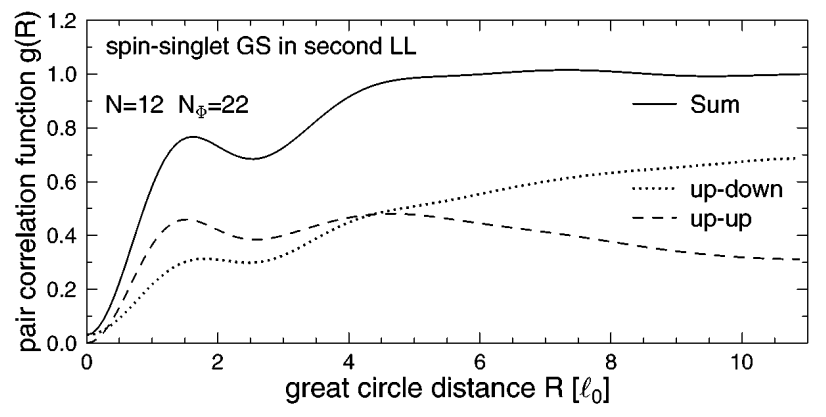

FIG. 2. Pair correlation function $g(R)$ of unpolarized GS for $N=12, N_{\Phi}=22$ in the second LL. 


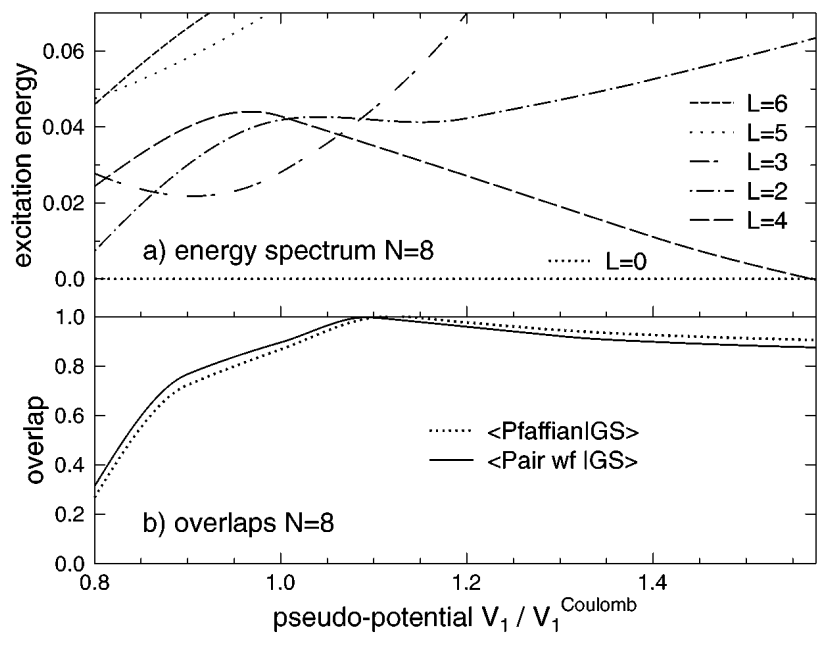

FIG. 3. (a) Energy of low-lying states of polarized system of 8 electrons in second LL at flux $N_{\Phi}=13$ vs the $L=1$ pseudopotential $V_{1}$, measured in units $V_{1}^{\text {Coulomb }}$. The state becomes gapless for small and large $V_{1}$. (b) Overlap of the GS wf with Pfaffian and pair-wf trial states. Gap and overlap both have their maximum at $V_{1} \approx 1.1$.

exhibit the characteristics of the Pfaffian, e.g., excitations with non-Abelian statistics[16].

In Fig. 4(a), we show the excitation spectrum for a much larger system, $N=16, N_{\Phi}=29$. The spectrum looks similar with a gap that vanishes when $V_{1}$ is below 0.9 or larger than 1.3. For Coulomb interaction, the gap is again $\Delta \approx 0.02$, and its maximum still occurs at $V_{1} \approx 1.1$. Similar excitation spectra are also seen for sizes $N=10$ and 14 , while the system with $N=12, N_{\Phi}=21$ is "aliased" [12] with a $\nu_{1}=3 / 5$ state and its interpretation as a $\nu_{1}=1 / 2$ state is dubious. The evidence for

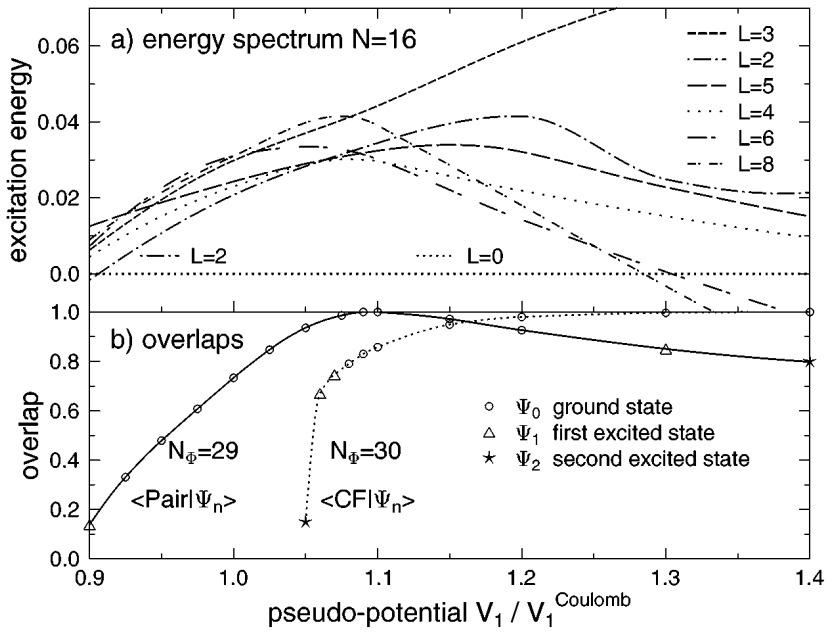

FIG. 4. (a) Same as Fig. 3(a) but for $N=16, N_{\Phi}=29$. This larger system becomes gapless too for small and large $V_{1}$. (b) Overlap of the GS $\Psi_{0}$ at $N=16, N_{\Phi}=30$ with the CF-liquid wf vs $V_{1}$ (dotted line). For large $V_{1}$, the overlap approaches unity. Overlap of the GS $\Psi_{0}$ at $N=16, N_{\Phi}=29$ with the "trial" state |pair $\rangle$, defined as GS for maximal gap (full line). phase transitions to gapless states for small and large $V_{1}$ appears firm.

The compressible state at large $V_{1}$ is the $\mathrm{CF}$ liquid [9,23]. This becomes clear from Fig. 4(b). At $N=16$, the CF state occurs at $N_{\Phi}=30$, one flux unit higher than for the $\mathrm{FQH}$ state. As a reference $\mathrm{CF}$ liquid wf, we use the GS for Coulomb interaction in the lowest LL [9]. As $V_{1}$ is increased, its overlap with the GS approaches unity when the system becomes gapless. As incompressible and CF states do not exist at the same flux $N_{\Phi}$, a bias exists in favor of the $\mathrm{FQH}$ state at $N_{\Phi}=29$, whereas the CF liquid is favored at $N_{\Phi}=30$. The critical $V_{1}$ value will thus be either overestimated or underestimated, depending on $N_{\Phi}$.

In Fig. 4(b), we also show the overlap of the GS $\Psi_{0}$ at $N=16, N_{\Phi}=29$ with a trial state |pair $\rangle$, which is the GS at $V_{1}=1.1$ where the gap is maximal. The rapid drop of the overlap $\left\langle\right.$ pair $\left.\mid \Psi_{0}\right\rangle$, as $V_{1}$ is reduced below one, very similar to the one observed for $\langle$ Pfaffian $\mid \mathrm{GS}\rangle$ at $N=8$ [cf. Fig. 3(b)], is another indicator for the phase transition to the compressible state at small $V_{1}$. This transition is associated with a small wave vector instability in the excitation spectrum. In our spherical system, it occurs at $L=2$ both for $N=8$ and 16 . This compressible state is not a CF liquid. It might be the charge density wave state proposed by Koulakov et al. [24]. To study such states, the torus geometry may be more appropriate.

It is instructive to study the system at the nearby $\nu=7 / 3$ filling since Hall plateaux at $5 / 2$ and $7 / 3$ have been observed in the same experiment [5]. The results for energy gap and GS overlap with the Laughlin state $\Psi_{3}$ shown in Fig. 5 are evidence for a phase transition from a gapless at small $V_{1}$ to an incompressible state at around $V_{1} \approx 0.96$. The energy gap for Coulomb interaction, $V_{1}=1$, is $\Delta_{7 / 3} \approx 0.02$ which is close to the calculated value at 5/2. In the activation studies of Eisenstein et al. [5], it was found that the gap at $\nu=7 / 3$ decreases with increasing tilt angle and disappears in much the same way as at $\nu=5 / 2$ [25]. As the FQH state at $7 / 3$ is almost certainly spin polarized, and according to our numerical results at $5 / 2$ likewise, a common origin for the reduction of the gaps with increasing tilt angle and for their disappearance may be expected. Our results imply that a reduction of $V_{1}$ would simultaneously reduce both gaps and eventually lead to compressible states. Besides increasing the Zeeman energy, which

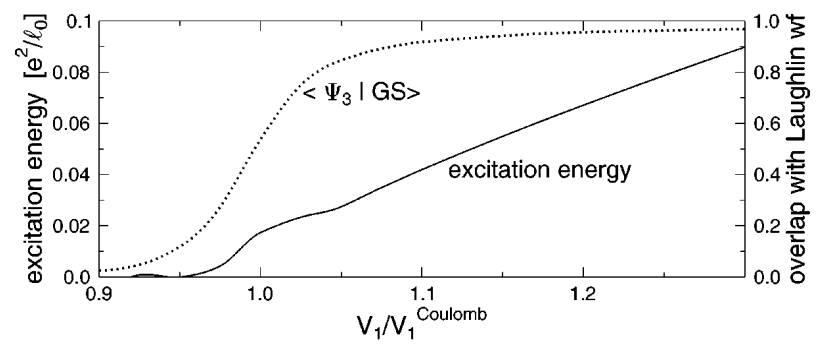

FIG. 5. Overlap of the GS with the Laughlin state $\Psi_{3}$ and excitation energy of 10 electron energy vs $V_{1}$ at $\nu=7 / 3$. 


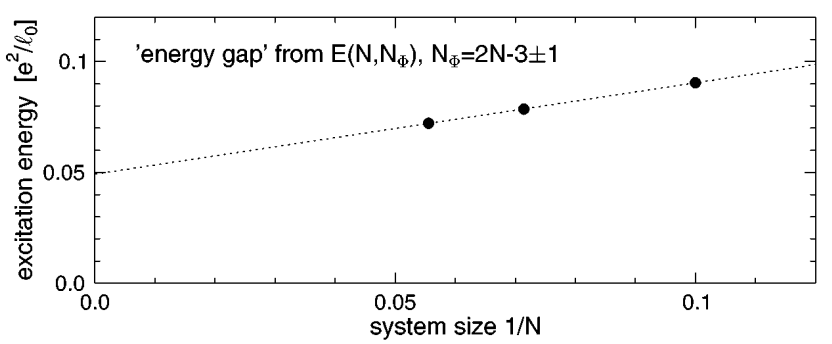

FIG. 6. Energy gap $\tilde{\Delta}$ of polarized system at $\nu_{1}=1 / 2$ vs system size $1 / N$, calculated from charged excitations.

cannot account for gap reduction in polarized states, a tilted $B$ field breaks rotational invariance in the plane and leads to a coupling between in-plane and perpendicular degrees of freedom, whose precise effect is not yet well understood. If the main effect of the in-plane $B$ field was a compression of the wf in the perpendicular direction, as is often assumed [3], an increase of $V_{1}$ [26] and a corresponding enhancement of the gap at $7 / 3$ would result, in conflict with experiment. But, if modifications to the electron interaction are indeed the cause for the gap reduction and the transition to compressible states at both $5 / 2$ and $7 / 3$, as we believe, a reduction of $V_{1}$ by just a few percent would suffice to explain the observed behavior.

Finally, in Fig. 6, we present the results of an alternative calculation of the gap from the energy of charged excitations, by changing the flux $N_{\Phi}$ by \pm 1 ; cf. [12]. Sizes not depicted are aliased by different types of FQH states and cannot be used for calculation of the gap [12]. The bulk limit $\tilde{\Delta}=0.050$, obtained by extrapolation in $1 / N$, is about twice the value $\Delta$ obtained above from neutral excitations. This is consistent with predictions [14,22] and with a GS for $S=2,4$ with angular momentum $L=$ $O\left(N^{0}\right)$, in which two quasiparticles are far apart to minimize their energy. At fixed field $B$, the gap $\Delta_{5 / 2}=0.025$ is about $1 / 4$ the gap $\Delta_{1 / 3}=0.102$ at $\nu=1 / 3$ [12], while at fixed density it is $\approx 11$ times smaller.

To conclude, our results imply that the " $g$ factor" determined from experiment [5] is not related to spin, but represents a correlation energy which should scale with $\sqrt{n_{S}}$. A detailed study of activation energies at $\nu=5 / 2$ and $7 / 3$ for samples of different densities together with a reliable calculation of tilted field effects will help decide if the key piece in the $\nu=5 / 2$ puzzle has now been found. But, the nature of the compressible state at small $V_{1}$ remains an open question.

I thank J. Dousson and A. Possoz for speeding up the computer code, and F. Schlepütz for providing a DECAlpha workstation with 1 Gbyte of memory.

I am grateful for stimulating discussions with $\mathrm{N}$. Cooper, D. Leadley, E. Rezayi, B. Shklovskii, R. Willett, and, in particular, with N. d'Ambrumenil, J. Eisenstein, B. I. Halperin, and H. Störmer who often encouraged me and helped improve the manuscript.
[1] R. Willett et al., Phys. Rev. Lett. 59, 1776 (1987).

[2] For a recent review of the quantum Hall effect, cf. Perspectives in Quantum Hall Effects, edited by S. Das Sarma and A. Pinczuk (Wiley-Interscience, New York, 1996).

[3] See the article by J.P. Eisenstein, in Perspectives in Quantum Hall Effects (Ref. [2]), p. 37.

[4] J. P. Eisenstein et al., Phys. Rev. Lett. 61, 997 (1988).

[5] J.P. Eisenstein et al., Surf. Sci. 229, 31 (1990).

[6] At small tilt angles, the gap at $8 / 5$ behaves similarly, cf. J. P. Eisenstein et al., Phys. Rev. Lett. 62, 1540 (1989).

[7] B. I. Halperin, P. A. Lee, and N. Read, Phys. Rev. B 47, 7312 (1993); cf. also B. I. Halperin, in Perspectives in Quantum Hall Effects (Ref. [2]), p. 225.

[8] See review by H.L. Störmer and D. C. Tsui, in Perspectives in Quantum Hall Effects (Ref. [2]), p. 385.

[9] R. Morf and N. d'Ambrumenil, Phys. Rev. Lett. 74, 5116 (1995).

[10] Partially polarized FQH states at $\nu_{1}=1 / 2$ cannot be ruled out a priori, but are not studied here.

[11] F. D. M. Haldane, Phys. Rev. Lett. 51, 605 (1983).

[12] N. d'Ambrumenil and R. Morf, Phys. Rev. B 40, 6108 (1989).

[13] X. G. Wen and A. Zee, Phys. Rev. Lett. 69, 953 (1992).

[14] F. D. M. Haldane and E. H. Rezayi, Phys. Rev. Lett. 60, 956 (1988); 60, 1886 (1988).

[15] If pairing of electrons plays a role, we are guided by the arguments of Ref. [14]: The flux of a paired state at $\nu=1 / 2$ is $N_{\Phi}=2 N-4+L_{\text {rel }}$, where $L_{\text {rel }}$ is the relative angular momentum of the electrons in a pair. Symmetry requires even (odd) $L_{\text {rel }}$ for spin-singlet (triplet) pairs. For $L_{\text {rel }}>2$ there is no pairing, as half-filling implies $L_{\text {rel }}=2$ in the mean, which leaves $S=2,4$ for spin-singlet and $S=3$ for polarized states as options.

[16] G. Moore and N. Read, Nucl. Phys. B360, 362 (1991).

[17] The finite width of the wf in the perpendicular direction modifies the interaction at short distance. However, the GS remains polarized and the qualitative conclusions of this work remain unchanged. R. Morf (to be published).

[18] For the second LL, we follow the mathematics of N. d'Ambrumenil and A. M. Reynolds, J. Phys. C 21, 119 (1988). The number of flux units $N_{\Phi}$ always refers to its value in the lowest LL. Details will be given elsewhere.

[19] R. Morf, N. d'Ambrumenil, and B. I. Halperin, Phys. Rev. B 34, 3037 (1986).

[20] That LL mixing effects are unlikely to change this may be inferred from recent results by N. R. Cooper, Phys. Rev. B 55, 1934 (1977), which show that around $\nu_{1}=1$, LL mixing favors polarized quasiparticles over Skyrmions.

[21] For $N \leq 10$, we verified that for the interaction, $V_{0}=$ $V_{2}=1$, and $V_{L}=0$ otherwise, zero energy unpolarized GS, with $g(0)=0$ in the second LL, exist if and only if $N_{\Phi}>3 N / 2$.

[22] M. Greiter, X.-G. Wen, and F. Wilczek, Phys. Rev. Lett. 66, 3205 (1991); M. Greiter, X.-G. Wen, and F. Wilczek, Nucl. Phys. B374, 567 (1992).

[23] E. Rezayi and N. Read, Phys. Rev. Lett. 72, 900 (1994).

[24] A. A. Koulakov, M. M. Fogler, and B. I. Shklovskii, Phys. Rev. Lett. 76, 499 (1996).

[25] J. P. Eisenstein (private communication).

[26] At $B=4 \mathrm{~T}$ and $n_{S}=2.3 \times 10^{11} \mathrm{~cm}^{-2}$ [5], characteristic wf width [17] of $7,10,20 \mathrm{~nm}$, lead to $V_{1} \approx$ $0.99,0.98,0.95$, respectively. 